JOURNAL OF

FUNCTION SPACES AND APPLICATIONS

Volume 3, Number 3 (2005), 223-237
(C) 2005, Scientific Horizon http://www.jfsa.net

\title{
A note on two-weight inequalities for multiple Hardy-type operators
}

\author{
Alexander Meskhi \\ (Communicated by Vakhtang Kokilashvili)
}

2000 Mathematics Subject Classification. 46B50, 46E40, 47B34, 47B38.

Keywords and phrases. Two-dimensional Hardy operators, two-weight inequality, the multiple Riemann-Liouville transforms, boundedness of operators.

Abstract. Necessary and sufficient conditions on a pair of weights guaranteeing two-weight estimates for the multiple Riemann-Liouville transforms are established provided that the weight on the right-hand side satisfies some additional conditions.

\section{Introduction}

In 1985 E. Sawyer [15] solved the two-weight problem for the twodimensional Hardy transform

$$
H_{2} f(x, y)=\int_{0}^{x} \int_{0}^{y} f(t, \tau) d t d \tau, \quad x, y>0 .
$$

Namely he proved the following statement:

Theorem A. Let $1<p \leq q<\infty$. Then for the boundedness of the operator $H_{2}$ from $L_{w}^{p}\left(R_{+}^{2}\right)$ to $L_{v}^{q}\left(R_{+}^{2}\right)$ it is necessary and sufficient that the following three independent conditions are satisfied: 
(i)

$$
A=: \sup _{a, b>0}\left(H_{2}^{\prime} v(a, b)\right)^{1 / q}\left(H_{2} \sigma(a, b)\right)^{1 / p^{\prime}}<\infty
$$

where $\sigma=: w^{1-p^{\prime}}, p^{\prime}=\frac{p}{p-1}$;

(ii)

$$
\int_{0}^{a} \int_{0}^{b}\left(H_{2} \sigma\right)^{q} v \leq A^{q}\left[H_{2} \sigma(a, b)\right]^{p / q}
$$

for all $a, b>0$;

(iii)

$$
\int_{a}^{\infty} \int_{b}^{\infty}\left(H_{2}^{\prime} v\right)^{p^{\prime}} \sigma \leq A^{p^{\prime}}\left[H_{2}^{\prime} v(a, b)\right]^{p^{\prime} / q^{\prime}}
$$

for all $a, b>0$, where

$$
H_{2}^{\prime} f(x, y)=\int_{x}^{\infty} \int_{y}^{\infty} f(t, \tau) d t d \tau, \quad x, y>0
$$

In her doctoral thesis A. Wedestig [20] derived a two-weight criterion for the operator $\mathrm{H}_{2}$ when the weight on the right-hand side is a product of two functions of separate variables. In particular, she proved

Theorem B. Let $1<p \leq q<\infty$ and let $s_{1}, s_{2} \in(1, p)$. Suppose that the weight function $w$ on $R_{+}^{2}$ has the form $w(x, y)=w_{1}(x) w_{2}(y)$. Then for the boundedness of the operator $\mathrm{H}_{2}$ from $L_{w}^{p}\left(R_{+}^{2}\right)$ to $L_{v}^{q}\left(R_{+}^{2}\right)$ it is necessary and sufficient that

$$
\begin{aligned}
A\left(s_{1}, s_{2}\right)=: & \sup _{t_{1}, t_{1}>0} W_{1}\left(t_{1}\right)^{\left(s_{1}-1\right) / p} W_{2}\left(t_{2}\right)^{\left(s_{2}-1\right) / p} \\
& \times\left(\int_{t_{1}}^{\infty} \int_{t_{2}}^{\infty} v\left(x_{1}, x_{2}\right) W_{1}\left(x_{1}\right)^{\frac{q}{p}\left(p-s_{2}\right)} d x_{1} d x_{2}\right)^{1 / q}<\infty,
\end{aligned}
$$

where $W_{1}\left(t_{1}\right)=\int_{0}^{t_{1}} w_{1}^{1-p^{\prime}}\left(x_{1}\right) d x_{1}$ and $W_{2}\left(t_{2}\right)=\int_{0}^{t_{2}} w_{2}^{1-p^{\prime}}\left(x_{2}\right) d x_{2}$.

Earlier some sufficient conditions for the validity of the two-weight inequality for $\mathrm{H}_{2}$ were established in [16] and [19].

Necessary and sufficient conditions on the weight function $v$ on $R_{+}^{2}$ governing the trace inequality

$$
\begin{aligned}
& \left(\int_{0}^{\infty} \int_{0}^{\infty}\left|R_{\alpha, \beta} f(x, y)\right|^{q} v(x, y) d x d y\right)^{1 / q} \\
& \quad \leq c\left(\int_{0}^{\infty} \int_{0}^{\infty}|f(x, y)|^{p} d x d y\right)^{1 / p}, 1<p \leq q<\infty
\end{aligned}
$$


for the Riemann-Liouville operator with multiple kernels

$$
R_{\alpha, \beta} f(x, y)=\int_{0}^{x} \int_{0}^{y} \frac{f(t, \tau)}{(x-t)^{1-\alpha}(y-\tau)^{1-\beta}} d t d \tau,
$$

where $\alpha, \beta>1 / p$, have been obtained in [8]. Analogous problem has been solved in [9] for $0<\alpha<1 / p$ and $\beta>1 / p$.

In this paper we establish boundedness criteria for the operator $R_{\alpha, \beta}$, $\alpha, \beta>1$, from $L_{w}^{p}\left(R_{+}^{2}\right)$ to $L_{v}^{q}\left(R_{+}^{2}\right)$ when the weight $w$ satisfies the onedimensional doubling condition uniformly with respect to another variable. As a corollary we conclude that under this restriction the two-weight inequality for the operator $H_{2}$ holds if and only if the condition (1.1) is satisfied. When the weight function $w$ has the form $w(x, y)=w_{1}(x) w_{2}(y)$ we show that also in this case a two-weight criterion for $H_{2}$ is (1.1).

\section{Preliminaries}

Let $\rho$ be an almost everywhere positive function on a subset $E$ of $R^{n}$. We denote by $L_{\rho}^{p}(E), 1<p<\infty$, the set of all measurable functions $f: E \rightarrow R^{1}$ for which the norm

$$
\|f\|_{L_{\rho}^{p}(E)}=\left(\int_{E}|f(x)|^{p} \rho(x) d x\right)^{1 / p}
$$

is finite.

Let us recall some well-known results for one-dimensional Hardy-type transforms.

A solution of the two-weight problem for the one-dimensional Hardy transform

$$
H f(x)=\int_{0}^{x} f(t) d t
$$

has been given by B. Muchenhoupt [13] for $p=q$; by V. Kokilashvili [6], J. Bradley [2] and V. Maz'ya [11, Chapter 1] for $p \leq q$. Namely the following statement holds.

Theorem C. Let $1<p \leq q<\infty$. Then the inequality

$$
\left(\int_{0}^{\infty}\left|\int_{0}^{x} f(y) d y\right|^{q} d x\right)^{1 / q} \leq c\left(\int_{0}^{\infty}|f(x)|^{p} d x\right)^{1 / p}
$$

with the positive constant $c$ independent of $f$ holds if and only if

$$
A \equiv \sup _{t>0}\left(\int_{t}^{\infty} v(x) d x\right)^{1 / q}\left(\int_{0}^{t} w^{1-p^{\prime}}(x) d x\right)^{1 / p^{\prime}}<\infty .
$$


Moreover, if $c$ is the best constant in (2.1), then there exists a positive constant $b$ depending only on $p$ and $q$ such that the inequality $A \leq c \leq b A$ holds.

Later on F. J. Martin-Reyes and E. Sawyer [10] and V. Stepanov [17] proved the next statement, which gives two-weight criteria for the RiemannLiouville transform

$$
R_{\alpha} f(x)=\int_{0}^{x} \frac{f(y)}{(x-y)^{1-\alpha}} d y
$$

where $\alpha>1$.

Theorem D. Let $1<p \leq q<\infty, \alpha>1$. Then the operator $R_{\alpha}$ acts boundedly from $L_{w}^{p}\left(R_{+}\right)$to $L_{v}^{q}\left(R_{+}\right)$if and only if the following two conditions

$$
\begin{aligned}
& A_{1}=: \sup _{t>0}\left(\int_{t}^{\infty} \frac{v(x)}{(x-t)^{(1-\alpha) q}} d x\right)^{1 / q}\left(\int_{0}^{t} w^{1-p^{\prime}}(x) d x\right)^{1 / p^{\prime}}<\infty ; \\
& A_{2}=: \sup _{t>0}\left(\int_{t}^{\infty} v(x) d x\right)^{1 / q}\left(\int_{0}^{t} \frac{w^{1-p^{\prime}}(x)}{(t-x)^{(1-\alpha) p^{\prime}}} d x\right)^{1 / p^{\prime}}<\infty
\end{aligned}
$$

hold. Moreover, there exist positive constants $c_{1}$ and $c_{2}$ depending only on $\alpha, p$ and $q$ such that $c_{1} \max \left\{A_{1}, A_{2}\right\} \leq\left\|R_{\alpha}\right\| \leq c_{2} \max \left\{A_{1}, A_{2}\right\}$.

Criteria for the boundedness of $R_{\alpha}$ from $L^{p}\left(R_{+}\right)$to $L_{v}^{q}\left(R_{+}\right)$when $1<p \leq q<\infty$ and $\alpha>1 / p$ have been obtained in [12] (see also [14]), while the similar result has been derived in [7], [3, Chapter 2], for $1<p \leq q<\infty$ and $0<\alpha<1 / p$. When $1<p<q<\infty$ a solution of the two-weight problem for potential operators has been given in [5].

The next statement concerning the discrete Hardy operator defined on $Z$ perhaps is already known, but we give the proof of the theorem for the completeness (see also [1], [4] for two-weight criteria for the Hardy transform on $\left.Z_{+}\right)$:

Theorem E. Let $1<p \leq q<\infty$ and let $\left\{a_{n}\right\},\left\{b_{n}\right\}$ be positive sequences. The inequality

$$
\left(\sum_{n=-\infty}^{\infty}\left|\sum_{k=-\infty}^{n} g_{k}\right|^{q} a_{n}^{q}\right)^{1 / q} \leq c\left(\sum_{n=-\infty}^{\infty}\left|g_{n}\right|^{p} b_{n}^{p}\right)^{1 / p}
$$


with the positive constant $c$ independent of $\left\{g_{k}\right\}\left(g_{k} \in l_{b_{n}^{p}}^{p}(Z)\right)$, holds if and only if

$$
B=: \sup _{n \in Z}\left(\sum_{k=n}^{\infty} a_{k}^{q}\right)^{1 / q}\left(\sum_{k=-\infty}^{n} b_{k}^{-p^{\prime}}\right)^{1 / p^{\prime}}<\infty
$$

Moreover, if $c$ is the best constant in (2.2), then

$$
B \leq c \leq B q^{\frac{1}{q}}\left(\frac{q}{q-1}\right)^{(p-1) / p}
$$

Proof (Sufficiency). Let $\alpha_{n}=\left(\sum_{k=-\infty}^{n} b_{k}^{-p^{\prime}}\right)^{\frac{1}{q p^{\prime}}}$. Due to Hölder's inequality we have

$$
\begin{aligned}
& \left(\sum_{n=-\infty}^{\infty} a_{n}^{q}\left|\sum_{k=-\infty}^{n} f_{k}\right|^{q}\right)^{p / q} \\
& \quad \leq\left(\sum_{n=-\infty}^{\infty} a_{n}^{q}\left(\sum_{k=-\infty}^{n}\left|f_{k} \alpha_{k} b_{k}\right|^{p}\right)^{q / p}\left(\sum_{k=-\infty}^{n}\left(\alpha_{k} b_{k}\right)^{-p^{\prime}}\right)^{\frac{q}{p^{\prime}}}\right)^{p / q} \\
& \quad=:\left(\sum_{n=-\infty}^{\infty} A_{n}\right)^{\frac{p}{q}} .
\end{aligned}
$$

By Minkowsky's inequality $\left(\frac{q}{p} \geq 1\right)$ we obtain

$$
\begin{aligned}
\left(\sum_{n=-\infty}^{\infty} A_{n}\right)^{p / q} & =\left(\sum_{n=-\infty}^{\infty}\left(\sum_{k=-\infty}^{n}\left|f_{k} \alpha_{k} b_{k}\right|^{p} a_{n}^{p}\left(\sum_{k=-\infty}^{n}\left(\alpha_{k} b_{k}\right)^{-p^{\prime}}\right)^{\frac{p}{p^{\prime}}}\right)^{\frac{q}{p}}\right)^{\frac{p}{q}} \\
& \leq \sum_{k=-\infty}^{\infty}\left|f_{k} \alpha_{k} b_{k}\right|^{p}\left(\sum_{n=k}^{\infty} a_{n}^{q}\left(\sum_{k=-\infty}^{n}\left(\alpha_{k} b_{k}\right)^{-p^{\prime}}\right)^{\frac{q}{p^{\prime}}}\right)^{\frac{p}{q}} \\
& =: \sum_{k} D_{k} .
\end{aligned}
$$

For the intrinsic sum we have

$$
\sum_{n=k}^{\infty} a_{n}^{q}\left(\sum_{k=-\infty}^{n}\left(\alpha_{k} b_{k}\right)^{-p^{\prime}}\right)^{\frac{q}{p^{\prime}}}=\sum_{n=k}^{\infty} a_{n}^{q}\left(\sum_{k=-\infty}^{n}\left(\sum_{i=-\infty}^{k} b_{k}^{-p^{\prime}}\right)^{-\frac{1}{q}} b_{k}^{-p^{\prime}}\right)^{\frac{q}{p^{\prime}}} .
$$


Moreover, the next easily verifiable inequality

$$
\sum_{k=-\infty}^{n} b_{k}^{-p^{\prime}}\left(\sum_{i=-\infty}^{k} b_{i}^{-p^{\prime}}\right)^{-\frac{1}{q}} \leq q^{\prime}\left(\sum_{k=-\infty}^{n} b_{k}^{-p^{\prime}}\right)^{1 / q^{\prime}}
$$

gives

$$
\sum_{n=k}^{\infty} a_{n}^{q}\left(\sum_{i=-\infty}^{n}\left(\alpha_{i} b_{i}\right)^{-p^{\prime}}\right)^{\frac{q}{p^{\prime}}}=\left(q^{\prime}\right)^{\frac{q}{p^{\prime}}} \sum_{n=k}^{\infty} a_{n}^{q}\left(\sum_{i=-\infty}^{n} b_{i}^{-p^{\prime}}\right)^{\frac{q}{q^{\prime} p^{\prime}}}
$$

Further, the latter sum does not exceed

$$
\lambda=B^{\frac{q}{q^{\prime}}}\left(q^{\prime}\right)^{\frac{q}{p^{\prime}}} \sum_{n=k}^{\infty} a_{n}^{q}\left(\sum_{i=n}^{\infty} a_{i}^{q}\right)^{-\frac{1}{q^{\prime}}}
$$

The inequality

$$
\sum_{n=k}^{\infty} a_{n}^{q}\left(\sum_{i=n}^{\infty} a_{i}^{q}\right)^{-\frac{1}{q^{\prime}}} \leq q\left(\sum_{i=k}^{\infty} a_{i}^{q}\right)^{1 / q}
$$

can also be easily verified. Therefore we obtain

$$
\begin{aligned}
\lambda & \leq q B^{\frac{q}{q^{\prime}}}\left(q^{\prime}\right)^{\frac{q}{p^{\prime}}}\left(\sum_{i=k}^{\infty} a_{i}^{q}\right)^{1 / q} \\
& \leq q B \cdot B^{\frac{q}{q^{\prime}}}\left(q^{\prime}\right)^{\frac{q}{p^{\prime}}}\left(\sum_{i=0}^{k} b_{i}^{-p^{\prime}}\right)^{-\frac{1}{p^{\prime}}} \\
& =q B^{q}\left(q^{\prime}\right)^{\frac{q}{p^{\prime}}} \alpha_{k}^{-q} .
\end{aligned}
$$

Finally we have

$$
\sum_{k \in Z} D_{k} \leq q^{\frac{p}{q}} B^{p}\left(q^{\prime}\right)^{\frac{p}{p^{\prime}}} \sum_{k=-\infty}^{\infty}\left|f_{k} \alpha_{k} b_{k}\right|^{p} \alpha_{k}^{-p}=q^{\frac{p}{q}} B^{p}\left(q^{\prime}\right)^{\frac{p}{p^{\prime}}} \sum_{k=-\infty}^{\infty}\left|f_{k}\right|^{p} \beta_{k}^{p}
$$

In order to prove necessity we take the sequence

$$
g_{k}= \begin{cases}\beta_{k}^{-p^{\prime}}, & k \leq n \\ 0, & k>n\end{cases}
$$


Then we have

$$
\left(\sum_{i \in Z}^{\infty}\left(\sum_{k=-\infty}^{i} g_{k}\right)^{q} a_{i}^{q}\right)^{1 / q} \geq\left(\sum_{i=n}^{\infty} a_{i}^{q}\right)^{1 / q}\left(\sum_{k=-\infty}^{n} \beta_{k}^{-p^{\prime}}\right) .
$$

On the other hand,

$$
\left(\sum_{k=-\infty}^{\infty}\left|g_{k}\right|^{p} \beta_{k}^{p}\right)^{1 / p}=\left(\sum_{k=-\infty}^{n} \beta_{k}^{-p^{\prime}}\right)^{1 / p}
$$

and finally

$$
B<\infty
$$

Analogously it follows

Theorem F. Let $1<p \leq q<\infty$ and let $m$ be an integer. Suppose that $\left\{a_{n}\right\}_{n=-\infty}^{m},\left\{b_{n}\right\}_{n=-\infty}^{m}$ are positive sequences. Then the two-weight inequality

$$
\left(\sum_{n=-\infty}^{m}\left|\sum_{k=-\infty}^{n} g_{k}\right|^{q} a_{n}^{q}\right)^{1 / q} \leq c\left(\sum_{n=-\infty}^{m}\left|g_{n}\right|^{p} b_{n}^{p}\right)^{1 / p}
$$

holds if and only if

$$
B_{m}=: \sup _{-\infty<n \leq m}\left(\sum_{k=n}^{m} a_{k}^{q}\right)^{1 / q}\left(\sum_{k=-\infty}^{n} b_{k}^{-p^{\prime}}\right)^{1 / p^{\prime}}<\infty .
$$

Moreover, if $c$ is the best constant in (2.3), then

$$
B_{m} \leq c \leq B_{m} q^{\frac{1}{q}}\left(\frac{q}{q-1}\right)^{(p-1) / p} .
$$

\section{The Main Results}

In order to formulate the main results of this paper we need the following definition:

Definition. A nonnegative function $\rho: R_{+}^{2} \rightarrow R^{1}$ is said to be a weight function with doubling condition uniformly with respect to $x \in R_{+}$if there exists a positive constant $c$ such that for arbitrary $t>0$ and almost all $x>0$ the inequality 


$$
\int_{0}^{2 t} \rho(x, y) d y \leq c \int_{0}^{t} \rho(x, y) d y
$$

holds. In this case we write $\rho \in D C(y)$. Analogously we define the class $D C(x)$.

Note that if the weight $\rho$ is integrable on $[0, a]^{2}, a>0$, then $\rho \in D C(y)$ is equivalent to the condition: there exists a constant $c>0$ such that for all intervals of finite length $I \subset R_{+}$and all $t>0$ the inequality

$$
\int_{I} \int_{0}^{2 t} \rho(x, y) d x d y \leq c \int_{I} \int_{0}^{t} \rho(x, y) d x d y
$$

holds.

Theorem 3.1. Let $1<p \leq q<\infty$ and let $\alpha, \beta \geq 1$. Suppose that $w^{1-p^{\prime}} \in D C(y)$. Then the operator $R_{\alpha, \beta}$ is bounded from $L_{w}^{p}\left(R_{+}^{2}\right)$ to $L_{v}^{q}\left(R_{+}^{2}\right)$ if and only if

(i)

$$
\begin{aligned}
A_{1}=: & \sup _{a, b>0}\left(\int_{0}^{a} \int_{0}^{b} \frac{w^{1-p^{\prime}}(x, y)}{(a-x)^{(1-\alpha) q}} d x d y\right)^{1 / p^{\prime}} \\
& \times\left(\int_{a}^{\infty} \int_{b}^{\infty} \frac{v(x, y)}{y^{(1-\beta) q}} d x d y\right)^{1 / q}<\infty
\end{aligned}
$$

(ii)

$$
\begin{aligned}
A_{2}=: & \sup _{a, b>0}\left(\int_{0}^{a} \int_{0}^{b} w^{1-p^{\prime}}(x, y) d x d y\right)^{1 / p^{\prime}} \\
& \times\left(\int_{a}^{\infty} \int_{b}^{\infty} \frac{v(x, y)}{(x-a)^{(1-\alpha) q} y^{(1-\beta) q}} d x d y\right)^{1 / q}<\infty
\end{aligned}
$$

Moreover, $\left\|R_{\alpha, \beta}\right\| \approx \max \left\{A_{1}, A_{2}\right\}$.

Theorem 3.2. Let $1<p \leq q<\infty$ and let $\alpha, \beta \geq 1$. Suppose that $w^{1-p^{\prime}} \in D C(x)$. Then the operator $R_{\alpha, \beta}$ is bounded from $L_{w}^{p}\left(R_{+}^{2}\right)$ to $L_{v}^{q}\left(R_{+}\right)$if and only if 
(i)

$$
\begin{aligned}
B_{1}=: & \sup _{a, b>0}\left(\int_{0}^{a} \int_{0}^{b} \frac{w^{1-p^{\prime}}(x, y)}{(b-y)^{(1-\beta) q}} d x d y\right)^{1 / p^{\prime}} \\
& \times\left(\int_{a}^{\infty} \int_{b}^{\infty} \frac{v(x, y)}{x^{(1-\alpha) q}} d x d y\right)^{1 / q}<\infty
\end{aligned}
$$

(ii)

$$
\begin{aligned}
B_{2}=: & \sup _{a, b>0}\left(\int_{0}^{a} \int_{0}^{b} w^{1-p^{\prime}}(x, y) d x d y\right)^{1 / p^{\prime}} \\
& \times\left(\int_{a}^{\infty} \int_{b}^{\infty} \frac{v(x, y)}{(y-b)^{(1-\beta) q} x^{(1-\alpha) q}} d x d y\right)^{1 / q}<\infty
\end{aligned}
$$

Moreover, $\left\|R_{\alpha, \beta}\right\| \approx \max \left\{B_{1}, B_{2}\right\}$.

Corollary 3.1. Let $1<p \leq q<\infty$. Suppose that $w^{1-p^{\prime}} \in D C(x)$ or $w^{1-p^{\prime}} \in D C(y)$. Then the operator $H_{2}$ is bounded from $L_{w}^{p}\left(R_{+}^{2}\right)$ to $L_{v}^{q}\left(R_{+}^{2}\right)$ if and only if (1.1) holds.

More general form of this corollary is the next statement:

Theorem 3.3. Let $1<p \leq q<\infty$. Assume that the weight function $w^{1-p^{\prime}}$ satisfies the condition

$$
\sup _{\substack{x>0 \\ k \in Z}}\left(\sum_{j=k}^{\infty}\left(\int_{0}^{2^{j}} w^{1-p^{\prime}}(x, y) d y\right)^{1-p}\right)\left(\int_{0}^{2^{k+1}} w^{1-p^{\prime}}(x, y) d x\right)^{p-1}<\infty .
$$

Then the boundedness of $H_{2}$ from $L_{w}^{p}\left(R_{+}^{2}\right)$ to $L_{v}^{q}\left(R_{+}^{2}\right)$ is equivalent to (1.1).

The following theorem states that if the weight function $w$ has the form $w(x, y)=w_{1}(x) w_{2}(y)$, then the boundedness of the operator $H_{2}$ from $L_{w}^{p}\left(R_{+}^{2}\right)$ to $L_{v}^{q}\left(R_{+}^{2}\right)$ is equivalent to the first condition in the E. Sawyer's theorem.

Theorem 3.4. Let $1<p \leq q<\infty$ and $w(x, y)=w_{1}(x) w_{2}(y)$. Then the operator $\mathrm{H}_{2}$ is bounded from $L_{w}^{p}\left(R_{+}^{2}\right)$ to $L_{v}^{q}\left(R_{+}^{2}\right)$ if and only if

$$
\begin{aligned}
D=: & \sup _{a, b>0}\left(\int_{0}^{a} w_{1}^{1-p^{\prime}}(x) d x\right)^{1 / p^{\prime}}\left(\int_{0}^{b} w_{2}^{1-p^{\prime}}(y) d y\right)^{1 / p^{\prime}} \\
& \times\left(\int_{a}^{\infty} \int_{b}^{\infty} v(x, y) d x d y\right)^{1 / q}<\infty
\end{aligned}
$$




\section{Proof of the Main Results}

In this section we present the proofs of the results formulated in the previous section.

Proof of Theorem 3.1. Sufficiency. First of all note that (see e.g., [18]) the condition $w^{1-p^{\prime}} \in D C(y)$ implies the reverse doubling condition for $w^{1-p^{\prime}}$ uniformly with respect to $x$, i.e., there exists the constants $\eta_{1}, \eta_{2}>1$ such that for all $t>0$ and a.e. $x \in R_{+}$the inequality

$$
\int_{0}^{\eta_{1} t} w^{1-p^{\prime}}(x, y) d y \geq \eta_{2} \int_{0}^{t} w^{1-p^{\prime}}(x, y) d y
$$

holds.

In the sequel we shall use the notation:

$$
\begin{gathered}
v_{1}(x, y)=: \frac{v(x, y)}{y^{(1-\beta) q}} ; \widetilde{v}_{1, j}(x)=: \int_{\eta_{1}^{j}}^{\eta_{1}^{j+1}} v_{1}(x, y) d y ; \\
F_{j}(t)=: \int_{0}^{\eta_{1}^{j+1}} f(t, \tau) d \tau ; \quad A=: \max \left\{A_{1}, A_{2}\right\} .
\end{gathered}
$$

Let $f \geq 0$. Then taking into account the fact $\alpha \geq 1$ and using Theorem $\mathrm{D}$ we find that

$$
\begin{aligned}
I & =: \int_{0}^{\infty} \int_{0}^{\infty} v(x, y)\left(R_{\alpha, \beta} f\right)^{q}(x, y) d x d y \\
& \leq \int_{0}^{\infty} \int_{0}^{\infty} v_{1}(x, y)\left(\int_{0}^{x}(x-t)^{\alpha-1}\left(\int_{0}^{y} f(t, \tau) d \tau\right) d t\right)^{q} d x d y \\
& \leq \sum_{j \in Z} \int_{0}^{\infty} \widetilde{v}_{1, j}(x)\left(\int_{0}^{x}(x-t)^{\alpha-1} F_{j}(t) d t\right)^{q} d x \\
& \leq c A^{q} \sum_{j \in Z}\left[\int_{0}^{\infty}\left(\int_{0}^{\eta_{1}^{j}} w^{1-p^{\prime}}(x, y) d y\right)^{1-p} F_{j}^{p}(x) d x\right]^{q / p} \\
& \leq c A^{q}\left[\int_{0}^{\infty} \sum_{j \in Z}\left(\int_{0}^{\eta_{1}^{j}} w^{1-p^{\prime}}(x, y) d y\right)^{1-p} F_{j}^{p}(x) d x\right]^{q / p} .
\end{aligned}
$$

On the other hand, we have

$$
\sup _{\substack{x>0 \\ k \in Z}} J(x, j)=: \sup _{\substack{x>0 \\ k \in Z}} \sum_{j=k}^{\infty}\left(\int_{0}^{\eta_{1}^{j}} w^{1-p^{\prime}}(x, y) d y\right)^{1-p}
$$




$$
\times\left(\sum_{j=-\infty}^{k} \int_{\eta_{1}^{j}}^{\eta_{1}^{j+1}} w^{1-p^{\prime}}(x, y) d y\right)^{p-1}<\infty .
$$

Indeed, (4.1) and the condition $w^{1-p^{\prime}} \in D C(y)$ lead to the inequality:

$$
\begin{aligned}
J(x, j)= & \sum_{j=k}^{\infty}\left(\int_{0}^{\eta_{1}^{j}} w^{1-p^{\prime}}(x, y) d y\right)^{1-p}\left(\int_{0}^{\eta_{1}^{k+1}} w^{1-p^{\prime}}(x, y) d y\right)^{p-1} \\
\leq & \left(\sum_{j=k}^{\infty} \eta_{2}^{(j-k)(1-p)}\right)\left(\int_{0}^{\eta_{1}^{k}} w^{1-p^{\prime}}(x, y) d y\right)^{1-p} \\
& \times\left(\int_{0}^{\eta_{1}^{k+1}} w^{1-p^{\prime}}(x, y) d y\right)^{p-1} \leq c .
\end{aligned}
$$

Consequently, by virtue of Theorem E and Hölder's inequality we find that

$$
\begin{aligned}
{\left[\int_{0}^{\infty} \sum_{j \in Z}\left(\int_{0}^{\eta_{1}^{j}} w^{1-p^{\prime}}(x, y) d y\right)^{1-p} F_{j}^{p}(x) d x\right]^{q / p} } \\
\leq c\left[\int_{0}^{\infty} \sum_{j \in Z}\left(\int_{0}^{\eta_{1}^{j}} w^{1-p^{\prime}}(x, y) d y\right)^{1-p}\left(\sum_{k=-\infty}^{j} \int_{\eta_{1}^{k}}^{\eta_{1}^{k+1}} f(x, t) d t\right)^{p} d x\right]^{q / p} \\
\leq c\left[\int_{0}^{\infty} \sum_{k \in Z}\left(\int_{\eta_{1}^{k}}^{\eta_{1}^{k+1}} w^{1-p^{\prime}}(x, \tau) d \tau\right)^{1-p}\left(\int_{\eta_{1}^{k}}^{\eta_{1}^{k+1}} f(x, t) d t\right)^{p} d x\right]^{q / p} \\
\leq c\left[\int_{0}^{\infty} \sum_{j \in Z}\left(\int_{\eta_{1}^{j}}^{\eta_{1}^{j+1}} w^{1-p^{\prime}}(x, y) d y\right)^{1-p}\right. \\
\left.\quad \times\left(\int_{\eta_{1}^{j}}^{\eta_{1}^{j+1}} w(x, t) f^{p}(x, t) d t\right)\left(\int_{\eta_{1}^{j}}^{\eta_{1}^{j+1}} w^{1-p^{\prime}}(x, t) d t\right)^{p-1} d x\right]^{q / p} \\
\leq c\|f\|_{L^{p}\left(R_{+}^{2}\right)}^{q} .
\end{aligned}
$$

Necessity. Let $f \geq 0$ and let $a, b>0$. It is easy to see that

$$
\begin{aligned}
I & \geq \int_{0}^{\infty} \int_{0}^{\infty} v(x, y)\left(\int_{0}^{x} \int_{0}^{y / 2} \frac{f(t, \tau)}{(x-t)^{1-\alpha}(y-\tau)^{1-\beta}} d t d \tau\right)^{q} d x d y \\
& \geq c\left(\int_{a}^{\infty} \int_{b}^{\infty} v_{1}(x, y) d x d y\right)\left(\int_{0}^{a} \int_{0}^{b / 2} \frac{f(t, \tau)}{(a-t)^{1-\alpha}} d t d \tau\right)^{q} .
\end{aligned}
$$

Using the latter inequality and the boundedness of $R_{\alpha, \beta}$ on the class of functions $f_{a, b}(x, y)=\chi_{(0, a)}(x) \chi_{(0, b / 2)}(y) w^{1-p^{\prime}}(x, y)(a-t)^{(\alpha-1)(q-1)}$, 
$a, b>0$, we find that

$$
\begin{gathered}
\left(\int_{0}^{a} \int_{0}^{b / 2} \frac{w^{1-p^{\prime}}(x, y)}{(a-x)^{(1-\alpha) q}} d x d y\right)^{q} \int_{a}^{\infty} \int_{b}^{\infty} v_{1}(x, y) d x d y \\
\leq I \leq c\left(\int_{0}^{a} \int_{0}^{b / 2} \frac{w^{1-p^{\prime}}(x, y)}{(a-x)^{(1-\alpha) q}} d x d y\right)^{q / p}<\infty
\end{gathered}
$$

Hence this inequality and the condition $w \in C D(y)$ give us the condition $A_{1}<\infty$.

Taking into account the arguments used above and the fact that the operator $R_{\alpha, \beta}$ is bounded from $L_{w}^{p}\left(R_{+}^{2}\right)$ to $L_{v}^{q}\left(R_{+}^{2}\right)$ if and only if its dual operator

$$
W_{\alpha, \beta} f(x, y)=\int_{x}^{\infty} \int_{y}^{\infty}(t-x)^{\alpha-1}(\tau-y)^{\beta-1} f(t, \tau) d t d \tau
$$

is bounded from $L_{v^{1-q^{\prime}}}^{q^{\prime}}\left(R_{+}^{2}\right)$ to $L_{w^{1-p^{\prime}}}^{p^{\prime}}\left(R_{+}^{2}\right)$, we obtain that $A_{2}<\infty$.

Proof of Theorem 3.2. The proof is similar to that of Theorem 3.1.

Proof of Theorem 3.3. Necessity is obvious. In order to prove sufficiency, it is enough to take the sequence $2^{k}$ instead of $\eta_{1}^{k}$ in the proof of Theorem 3.1.

Proof of Theorem 3.4. First suppose that $S:=\int_{0}^{\infty} w_{2}^{1-p^{\prime}}(y) d y=\infty$. Let $\left\{x_{k}\right\}_{k=-\infty}^{+\infty}$ be a sequence of positive numbers for which the equality

$$
2^{k}=\int_{0}^{x_{k}} w_{2}^{1-p^{\prime}}(y) d y
$$

holds for all $k \in Z$. It is clear that $\left\{x_{k}\right\}$ is increasing and $R_{+}=$ $\cup_{k \in Z}\left[x_{k}, x_{k+1}\right)$. Besides, it is easy to verify that

$$
2^{k}=\int_{x_{k}}^{x_{k+1}} w_{2}^{1-p^{\prime}}(y) d y
$$

Let $f \geq 0$. We have

$$
\begin{aligned}
I & =: \int_{0}^{\infty} \int_{0}^{\infty} v(x, y)\left(H_{2}(x, y)\right)^{2} d x d y \\
& =\sum_{k \in Z} \int_{0}^{\infty} \int_{x_{k}}^{x_{k+1}} v(x, y)\left(\int_{0}^{x} \int_{0}^{y} f(t, \tau) d t d \tau\right)^{q} d x d y \\
& \leq \sum_{k \in Z} \int_{0}^{\infty}\left(\int_{x_{k}}^{x_{k+1}} v(x, y) d y\right)\left(\int_{0}^{x}\left(\int_{0}^{x_{k+1}} f(t, \tau) d \tau\right) d t\right)^{q} d x
\end{aligned}
$$




$$
=\sum_{k \in Z} \int_{0}^{\infty} V_{k}(x)\left(\int_{0}^{x} F_{k}(t) d t\right)^{q} d x
$$

where

$$
V_{k}(x)=: \int_{x_{k}}^{x_{k+1}} v(x, y) d y ; \quad F_{k}(t)=: \int_{0}^{x_{k+1}} f(t, \tau) d \tau .
$$

Further, it is obvious that

$$
D^{q} \geq \sup _{\substack{a>0 \\ j \in Z}}\left(\int_{a}^{\infty} \int_{x_{j}}^{x_{j+1}} v(x, y) d x d y\right)\left(\int_{0}^{a} \int_{0}^{x_{j}} w^{1-p^{\prime}}(x, y) d x d y\right)^{q / p^{\prime}} .
$$

Therefore by Theorem $\mathrm{C}$ we obtain

$$
\begin{aligned}
I \leq & c D^{q} \sum_{j \in Z}\left[\int_{0}^{\infty} w_{1}(x)\left(\int_{0}^{x_{j}} w_{2}^{1-p^{\prime}}(y) d y\right)^{1-p}\left(F_{k}(x)\right)^{p} d x\right]^{q / p} \\
\leq & c D^{q}\left[\int_{0}^{\infty} w_{1}(x) \sum_{j \in Z}\left(\int_{0}^{x_{j}} w_{2}^{1-p^{\prime}}(y) d y\right)^{1-p}\right. \\
& \left.\times\left(\sum_{k=-\infty}^{j} \int_{x_{k}}^{x_{k+1}} f(x, \tau) d \tau\right)^{p} d x\right]^{q / p} .
\end{aligned}
$$

On the other hand, (4.3) yields

$$
\begin{aligned}
& \sum_{k=n}^{+\infty}\left(\int_{0}^{x_{k}} w_{2}^{1-p^{\prime}}(y) d y\right)^{1-p}\left(\sum_{k=-\infty}^{n} \int_{x_{k}}^{x_{k+1}} w_{2}^{1-p^{\prime}}(y) d y\right)^{p-1} \\
& \quad=\sum_{k=n}^{+\infty}\left(\int_{0}^{x_{k}} w_{2}^{1-p^{\prime}}(y) d y\right)^{1-p}\left(\int_{0}^{x_{n+1}} w_{2}^{1-p^{\prime}}(y) d y\right)^{p-1} \\
& =\left(\sum_{k=n}^{+\infty} 2^{k(1-p)}\right) 2^{(n+1)(p-1)} \leq c
\end{aligned}
$$

for all $n \in Z$. Hence by Theorem $\mathrm{E}$ and Hölder's inequality we have

$$
\begin{aligned}
I \leq & c D^{q}\left[\int_{0}^{\infty} w_{1}(x) \sum_{j \in Z}\left(\int_{x_{j}}^{x_{j+1}} w_{2}^{1-p^{\prime}}(y) d y\right)^{1-p}\right. \\
& \left.\times\left(\int_{x_{k}}^{x_{k+1}} f(x, \tau) d \tau\right)^{p} d x\right]^{q / p} \\
\leq & c D^{q}\left[\int_{0}^{\infty} w_{1}(x) \sum_{j \in Z}\left(\int_{x_{k}}^{x_{k+1}} w_{2}(\tau) f^{p}(x, \tau) d \tau\right) d x\right]^{q / p}=c D^{q}\|f\|_{L^{p}\left(R_{+}^{2}\right)}^{q} .
\end{aligned}
$$


If $S<\infty$, then without loss of generality we can assume that $S=1$. In this case we choose the sequence $\left\{x_{k}\right\}_{k=-\infty}^{0}$ for which (4.3) holds for all $k \leq 0$. Arguing as in the case $S=\infty$ and using Theorem F instead of Theorem E, we finally obtain the desired result.

Acknowledgements. It is a great pleasure for me to expresses my gratitude to Professor Vakhtang Kokilashvili for drawing the author's attention to the problems studied above and for helpful remarks and suggestions.

\section{References}

[1] G. Bennett, Some elementary inequalities, Quart. J. Math. Oxford, 38 (2) (1987), 401-425.

[2] J. S. Bradley, Hardy inequality with mixed norms, Canad. Math. Bull., 21 (1978), 405-408.

[3] D. E. Edmunds, V. Kokilashvili and A. Meskhi, Bounded and compact integral operators, Kluwer Academic Publishers, Dordrecht, Boston, London, 2002.

[4] I. Gabisonija and A. Meskhi, Two-weighted inequalities for a discrete Hilbert transform, Proc. A. Razmadze Math. Inst., 116 (1998), 107122.

[5] I. Genebashvili, A. Gogatishvili, V. Kokilashvili and M. Krbec, Weight theory for integral transforms on spaces of homogeneous type, Pitman Monographs and Surveys in Pure and Applied Mathematics, 92 (1998), Longman, Harlow.

[6] V. M. Kokilashvili, On Hardy's inequalities in weighted spaces (Russian), Soobsch. Akad. Nauk Gruz. SSR, 96 (1979), 37-40.

[7] V. Kokilashvili and A. Meskhi, On a trace inequality for one-sided potentials and applications to the solvability of nonlinear integral equations, Georgian Math. J., 8 (3) (2001), 521-536.

[8] V. Kokilashvili and A. Meskhi, On one-sided potentials with multiple kernels, Integral Transf. Spec. Functions, accepted for publication.

[9] V. Kokilashvili and A. Meskhi, On a trace inequality for one-sided potentials with multiple kernels, Frac. Calc. Appl. Anal., 6 (4) (2003), 461-472.

[10] F. J. Martin-Reyes and E. Sawyer, Weighted inequalities for RiemannLiouville fractional integrals of order one and greater, Proc. Amer. Math. Soc., 106 (1989), 727-733.

[11] V. G. Maz'ya, Sobolev Spaces, Springer, Berlin, 1985. 
[12] A. Meskhi, Solution of some weight problems for the Riemann-Liouville and Weyl operators, Georgian Math. J., 5 (6) (1998), 565-574.

[13] B. Muckenhoupt, Hardy's inequality with weights, Studia Math., 44 (1972), 31-38.

[14] D. V. Prokhorov, On the boundedness of a class of integral operators, J. London Math. Soc., 61 (2) (2000), 617-628.

[15] E. Sawyer, Weighted inequalities for the two-dimensional Hardy operator, Studia Math., 82 (1985), 1-11.

[16] V. N. Sedov, Weighted spaces, The imbedding theorem (Russian), Diff. Uravneniya, 8 (1972), 1452-1462.

[17] V. D. Stepanov, Weighted inequalities of Hardy type for higher derivatives, and their applications (Russian), Soviet Math. Dokl., 38 (1989), 389-393.

[18] J. O. Strömberg and A. Torchinsky, Weighted Hardy Spaces, Lecture Notes in Math., 1381, Springer Verlag, Berlin, 1989.

[19] F. A. Sysoeva, Generalization of a certain Hardy inequality (Russian), Izv. Vusch. Uchev. Zaved. Matematika, 49 (6) (1965), 140-143.

[20] A. Wedestig, Weighted inequalities of Hardy-type and their limiting inequalities, Doctoral Thesis, 2003.

A. Razmadze Mathematical Institute

Georgian Academy of Sciences

1. M. Aleksidze St.

0193 Tbilisi

Georgia

and

Scuola Normale Superiore

Piazza Dei Cavalieri 7

56126 Pisa

Italy

(E-mails : a.meskhi@sns.it, meskhi@rmi.acnet.ge)

(Received : August 2004) 


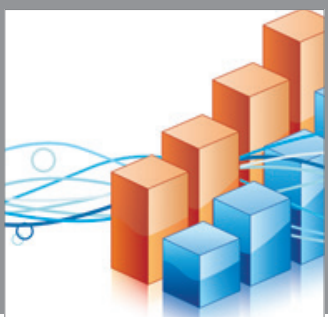

Advances in

Operations Research

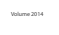

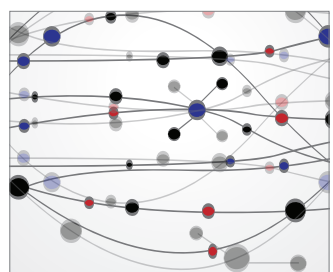

\section{The Scientific} World Journal
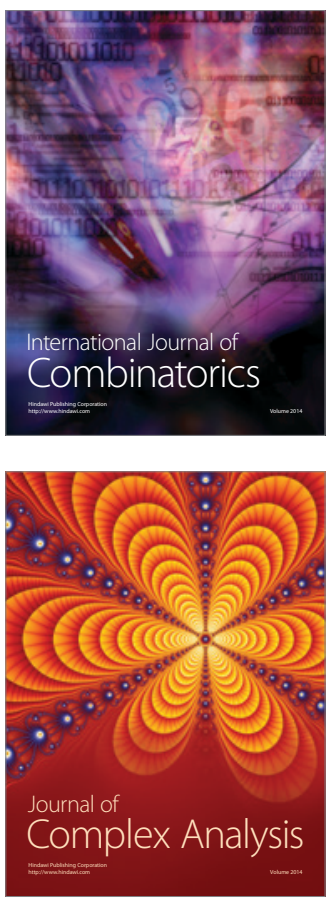

International Journal of

Mathematics and

Mathematical

Sciences
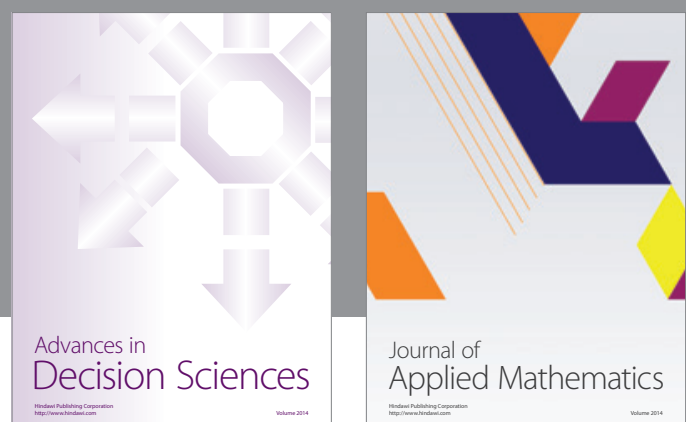

Journal of

Applied Mathematics
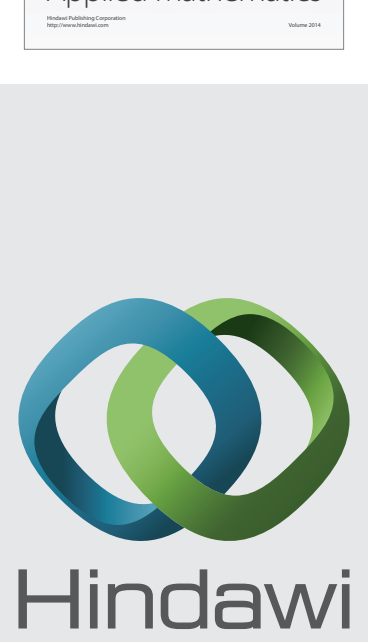

Submit your manuscripts at http://www.hindawi.com
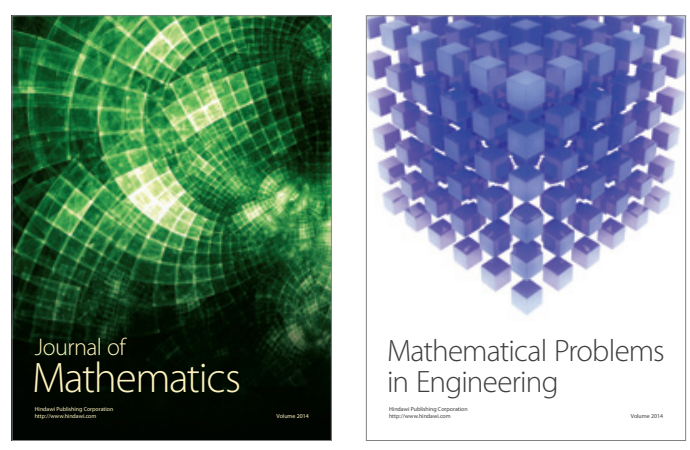

Mathematical Problems in Engineering
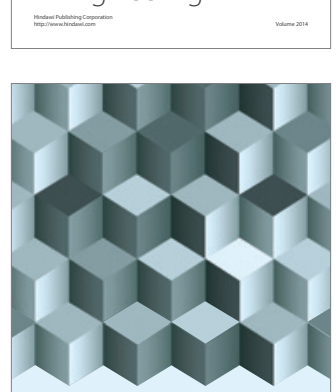

Journal of

Function Spaces
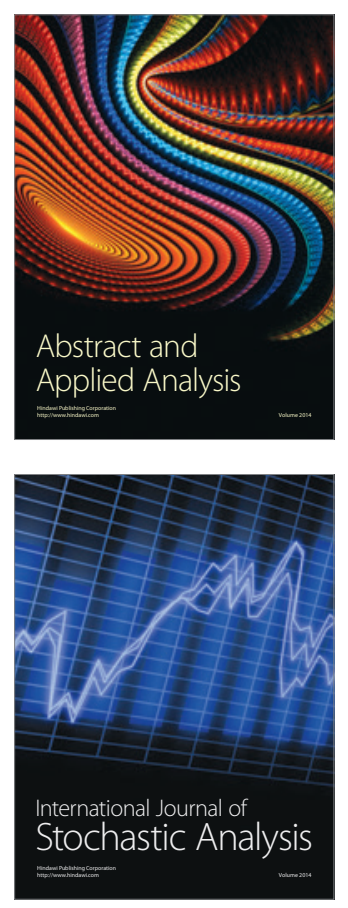

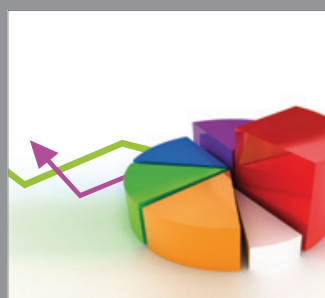

ournal of

Probability and Statistics

Promensencen
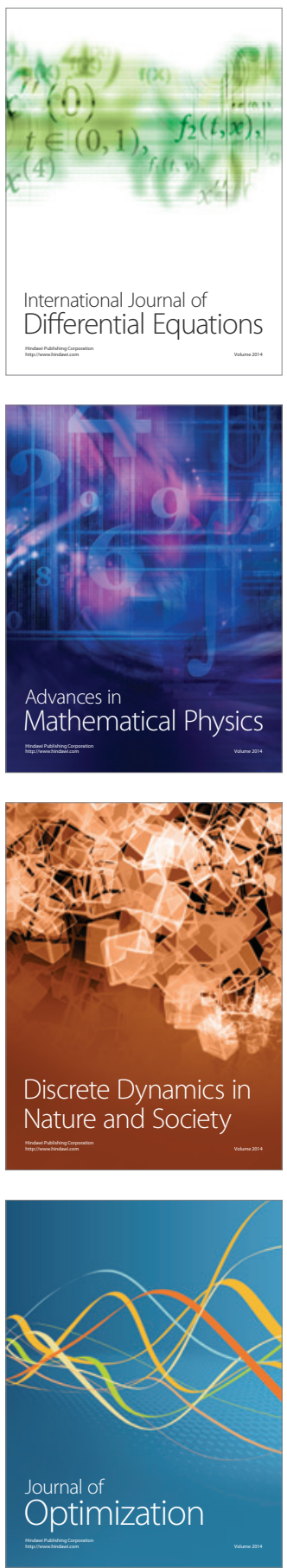